\title{
ASYMPTOTIC CONVERGENCE OF OPERATORS IN HILBERT SPACE ${ }^{1}$
}

\author{
FRANK GILFEATHER
}

The purpose of this paper is to study the strong convergence of the sequence $\left\{A^{n}\right\}$, where $A$ is an operator on a Hilbert space (cf. [3], [6]). It is known that if $A$ is a completely nonunitary contraction ${ }^{2}$ on a Hilbert space, then the sequence $\left\{A^{n}\right\}$ converges weakly. The question naturally arises as to which operators on a Hilbert space does the weak convergence of $\left\{A^{n}\right\}$ imply the strong convergence of $\left\{A^{n}\right\}$. In $\S 2$, we investigate this question for completely continuous operators and spectral operators with completely continuous imaginary parts.

Indeed, we will prove that $\left\{A^{n}\right\}$ converges strongly for certain operators $A$ using the weak convergence of the sequence $\left\{A^{n}\right\}$ and certain spectral properties of $A$. It seems that this approach to the strong convergence of $\left\{A^{n}\right\}$ is new and essentially different from the methods already employed. F. Browder and W. Petryshyn have shown that for a selfadjoint contraction $A$, the sequence $\left\{A^{n}\right\}$ converges strongly if and only if $(-1)$ is not a proper value of $A[3$, Theorem 3]. Our work will generalize this result. In connection with the functional equation $x-A x=y$ in Hilbert space, Browder and Petryshyn have shown that if $\left\{A^{n}\right\}$ converges strongly then solutions of this equation can be found. In $\S 3$ a result similar to this is obtained if $\left\{A^{n}\right\}$ converges weakly.

Throughout this paper an operator always means a bounded linear transformation on a complex Hilbert space $H$. An operator is said to be strong (resp. weak) asymptotically convergent if the sequence $\left\{A^{n}\right\}$ converges strongly (resp. weakly) in the space $\mathscr{L}(H)$ of all bounded operators on $H$. For an operator $A$ on $H$, if there exists an integer $M<\infty$ such that $\left\|A^{n}\right\|<M, n=1,2, \cdots$, then $A$ is said to be asymptotically bounded. If $M \leqq 1$ then $A$ is called a contraction. We will denote by $\Re(A)$ the null set of the operator $A$, i.e. $\Re(A)$ $=\{x \in H \mid A(x)=0\}$.

1. This section is concerned with the asymptotic convergence of a

Received by the editors August 2, 1968.

1 This work is part of the author's Ph.D. dissertaion at the University of California, Irvine, directed by Professor Noboru Suzuki.

${ }^{2}$ An operator $A$ is said to be completely nonunitary (resp. selfadjoint, normal) if $A$ does not have a nonzero reducing subspace $\mathfrak{M}$ such that $A / \mathfrak{M}$ is unitary (resp. selfadjoint, normal). 
general contraction. Our result is based on the following lemma which is a consequence of [9, Theorem 2] (for direct proofs, see [1] and [5]).

LEMMA 1. Every completely nonunitary contraction is weak asymptotically convergent to zero.

In what follows, $D$ will denote the set $\{-1,1\}$ union the interior of the unit disk in the complex plane. By decomposing a contraction into unitary and completely nonunitary parts we can prove:

Theorem 1. Let $A$ be a contraction such that the spectrum of $A$, denoted by $\sigma(A)$, is contained in $D$. Then $A$ is weak asymptotically convergent if and only if $(-1)$ is not a proper value of $A$.

Proof. There exists a projection $P$ commuting with $A$ such that $A=A_{P} \oplus A_{I-P}$ where $A_{P}$, the restriction of $A$ to $P H$, is unitary and $A_{I-P}$, the restriction of $A$ to $(I-P) H$, is completely nonunitary (cf. [8, Theorem 3.2, p. 9]). Since $\sigma(A)=\sigma\left(A_{P}\right) \cup \sigma\left(A_{I-P}\right)$ and $A_{P}$ is unitary, thus $\sigma\left(A_{P}\right) \subset\{-1,1\}$. Suppose that $(-1)$ is not a proper value of $A$. Then $\sigma\left(A_{P}\right)=\{1\}$ because isolated points of the spectrum of unitary operators are proper values. Thus $A_{P}$ is the identity $I_{P}$ on $P H$. Clearly, $\left\{A_{P}^{n}\right\}$ converges to $I_{P}$ on $P H$, and by Lemma $1,\left\{A_{I-P}^{n}\right\}$ converges weakly to zero on $(I-P) H$. Therefore $\left\{A^{n}\right\}$ converges weakly to $I_{P} \oplus 0$, that is to say, $A$ is weak asymptotically convergent.

Conversely, it should be noted that for any operator to be weak or strong asymptotically convergent, it is necessary that $\lambda$ is not a proper value of $A$ if $|\lambda| \geqq 1$ and $\lambda \neq 1$.

Remark. Let $A$ be a contraction on $H$. Because of the decomposition $H=\Re(I-A) \oplus(I-A) H^{-}$(cf. $\left.[10, \S 144]\right)$, if $\left\{A^{n}\right\}$ converges weakly or strongly then the limit is just the projection on $\Re(I-A)$. Furthermore, $\mathfrak{N}(I-A)$ and $(I-A) H^{-}$are reducing subspaces for $A$.

2. In this section we restrict our attention to the cases of spectral operators with completely continuous imaginary parts and completely continuous operators. It will be shown that the spectral conditions in Theorem 1 imply the strong asymptotic convergence of the operator. If, in fact $\left\{S^{n}\right\}$ does converge and has completely continuous imaginary part we can show $S$ has the spectral conditions of Theorem 1.

LEMMa 2. Let $S$ be a strong asymptotically convergent operator with completely continuous imaginary part. Then $\sigma(S) \subset D$ and $(-1)$ is not a proper value of $S$.

Proof. By the Principle of Uniform Boundedness, $\left\|S^{n}\right\| \leqq M<\infty$ for $n=1,2, \cdots$. Let $\rho(S)$ be the spectral radius of $S$ then $\rho(S)$ 
$=\lim _{n}\left\|S^{n}\right\| 1 / n \leqq \lim _{n} M^{1 / n}=1$. Thus $\lambda \in \sigma(S)$ implies that $|\lambda| \leqq 1$. Because of the fact that $S$ has completely continuous imaginary part, every nonreal element of $\sigma(S)$ is a proper value of $S$. As in the 'necessary' part of the proof of Theorem 1, we can conclude that $\sigma(S) \subset D$ and $(-1)$ is not a proper value of $S$.

If $A$ is an operator and $\left\{A^{n}\right\}$ converges strongly then $\left\{A^{n}\right\}$ always converges weakly. In particular, if $A$ is a selfadjoint contraction, then the converse is also true.

Lemma 3. Let $A$ be a selfadjoint contraction. Then $A$ is strong asymptotically convergent if and only if $A$ is weak asymptotically convergent.

Proof. By the remark in $\S 1, H=\mathfrak{N}(I-A) \oplus(I-A) H^{-}$where $\Re(I-A)$ and $(I-A) H$-reduce $A$. If we set $A_{1}=A / \Re(I-A)$ and $A_{2}=A /(I-A) H^{-}$, then $A=A_{1} \oplus A_{2}$. Clearly $\left\{A_{1}^{n}\right\}$ converges strongly. For $x \in(I-A) H^{-}$we have $\left\|A_{2}^{n} x\right\|^{2}=\left\langle A_{2}^{n}(x), A_{2}^{n}(x)\right\rangle=\left\langle A_{2}^{2 n} x, x\right\rangle$ $\rightarrow 0$ as $n \rightarrow \infty$. Thus $A$ is strong asymptotically convergent.

In the following two theorems we will make use of the technical lemma:

LeMma 4. Let $\left\{A_{n}\right\}$ be a sequence of operators on $H$ with uniformly bounded norms which converges strongly to an operator $B$ on a dense set in $H$. Then $\left\{A_{n}\right\}$ converges strongly to $B$ on $H$.

Proof. The lemma follows from the inequalities:

$$
\begin{aligned}
\left\|A_{n} x-B x\right\| & \leqq\left\|A_{n} x-A_{n} y\right\|+\left\|A_{n} y-B y\right\|+\|B y-B x\| \\
& \leqq(M+\|B\|)\|x-y\|+\left\|A_{n} y-B y\right\|,
\end{aligned}
$$

where $\left\|A_{n}\right\| \leqq M$ for $n=1,2, \cdots$ and $x, y \in H$.

As operator $A$ on $H$ is called a spectral operator if there exists a resolution of the identity for $A$. If $E$ is a resolution of the identity for an operator $A$ and $A=\int_{\Omega} \lambda d E \lambda$, where $\Omega$ is the support of $E$ and is compact, then $A$ is called a scalar type operator. It has been shown in [4] that an operator $S$ is of scalar type if and only if $S$ $=R T R^{-1}$ where $T$ is a normal operator and $R$ is an invertible operator on $H$. Furthermore, N. Dunford has shown that $T$ is a spectral operator on $H$ if and only if $T=S+N$ where $S$ is of scalar type, $N$ is a quasinilpotent operator and $S N=N S$. The decomposition $T=S+N$ of a spectral operator is unique. For these statements and the details of the theory of spectral operators we refer to [4].

THEOREM 2. Let $S$ be a scalar type operator with completely continuous imaginary part. Then $S$ is strong asymptotically convergent if and only if $\sigma(S) \subset D$ and $(-1)$ is not a proper value of $S$. 
Before proving this theorem let us show that we need only consider the case when $S$ is a normal operator with completely continuous imaginary part. Since $S$ is a scalar type operator, there exists an invertible operator $R$ such that $R S R^{-1}=T$ and $T$ is normal. Furthermore, N. Suzuki has pointed out in [13] that if $S$ has completely continuous imaginary part then so does $T$. For completeness we shall sketch the proof of that statement. The spectral properties of an operator with completely continuous imaginary part are well known (cf. [2]). Since $T$ is similar to $S$ we can conclude that every nonreal point in $\sigma(T)$ is an isolated point, that there are at most countably many of them, which we denote by $\left\{\lambda_{n}\right\}$, and if the set is infinite, then $\operatorname{Im} \lambda_{n} \rightarrow 0$. Furthermore, if $\lambda_{i}$ is a nonreal point in $\sigma(S)$, then the proper subspace $\Re\left(\lambda_{i}-S\right)$ is finite dimensional. Since $\Re\left(\lambda_{i}-S\right)$ $=R \Re\left(\lambda_{i}-T\right)$, it follows that $\Re\left(\lambda_{i}-T\right)$ is finite dimensional. Thus we can decompose $T$ as $T_{0} \oplus \sum \lambda_{i} I_{i}$ where $H=H_{0} \oplus \sum \oplus \Re\left(\lambda_{i}-T\right)$. Since $T_{0}$ is normal with real spectrum we conclude that $T_{0}$ is selfadjoint. It then follows that $T$ has completely continuous imaginary part.

The sequence $\left\{S^{n}\right\}$ converges strongly if and only if $\left\{T^{n}\right\}$ converges strongly and $T$ satisfies the conditions $\sigma(T) \subset D$ with $(-1)$ not a proper value of $T$ if and only if $S$ satisfies these conditions for $S$. Thus to prove Theorem 2, we can assume that $S$ is normal.

Proof of Theorem 2. There exists a projection $P$ commuting with $S$ such that $S=S_{P} \oplus S_{I-P}$ where $S_{P}$, the restriction of $S$ to $P H$, is selfadjoint and $S_{I-P}$, the restriction of $S$ to $(I-P) H$, is completely nonselfadjoint. Furthermore, $(I-P) H$ is generated by vectors of the form $S^{n} C x$, where $x \in H, n=0,1,2, \cdots$, and $C=\operatorname{Im}(S)=\left(S-S^{*}\right) / 2 i$ (cf. [2, p. 279]). Since $C$ commutes with $S$, therefore $C(H)^{-}=(I-P) H$.

Suppose that $\sigma(S) \subset D$ and $(-1)$ is not a proper value of $S$. Since $\rho(S)=\|S\|$ then $\|S\| \leqq 1$ and $S$ is a contraction. By Theorem $1,\left\{S^{n}\right\}$ converges weakly and in particular $\left\{S_{P}^{n}\right\}$ and $\left\{S_{I-P}^{n}\right\}$ converge weakly. $S_{P}$ is selfadjoint, thus Lemma 3 implies that $\left\{S_{P}^{n}\right\}$ converges strongly. If $x \in C(H), x=C y$ for some $y \in H$, then $\left(S_{I-P}\right)^{n} x=\left(S_{I-P}\right)^{n} C y$ $=C\left(S_{I-P}\right)^{n} y$. Because $\left\{\left(S_{I-P}\right)^{n} y\right\}$ converges weakly and $C$ transforms weak convergent sequences into strong convergent sequences, $\left\{\left(S_{I-P}\right)^{n} x\right\}$ converges strongly. By Lemma $4, \quad\left\{S^{n}\right\}$ converges strongly on $(I-P) H$. Therefore $S$ is strong asymptotically convergent.

Conversely, let $\left\{S^{n}\right\}$ converge strongly. Then by Lemma 2, we can conclude that $\sigma(S) \subset D$ and $(-1)$ is not a proper value of $S$.

REMARK. Theorem 2 directly generalizes [3, Theorem 3], as mentioned in the introduction. In that theorem if $S$ is a selfadjoint con- 
traction then $\left\{S^{n}\right\}$ converges strongly if and only if $(-1)$ is not a proper value of $S$. This result follows directly from Theorem 1 and Lemma 3.

We now extend Theorem 2 to the case where $S$ is a spectral contraction.

THEOREM 3. Let $A$ be a spectral contraction with completely continuous imaginary part. Then $A$ is strong asymptotically convergent if and only if $\sigma(A) \subset D$ and $(-1)$ is not a proper value of $A$.

Proof. Let $A=S+N$ be the canonical decomposition of $A$ into scalar and radical parts. By Theorem 1 in [12] $N$ is completely continuous, so that $\operatorname{Im}(S)=\operatorname{Im}(A)-\operatorname{Im}(N)$ is completely continuous. Let $R$ be an invertible operator such that $R S R^{-1}=T$ and $T$ is a normal operator. Then as in Theorem $2, T$ has completely continuous imaginary part.

Let $B=R A R^{-1}=R S R^{-1}+R N R^{-1}=T+L$. Then $T$ is normal, $L$ is quasinilpotent and completely continuous and $T L=L T$. The operators $B, A, S$ and $T$ all have the same spectrum. The following decomposition for $B$ is given in [12]. The Hilbert space $H=\sum_{i=0}^{n} \oplus H_{i}$, $n \leqq \infty$, and $B=T / H_{0} \oplus \sum_{i=1}^{n} \oplus \lambda_{i}+L_{i}$, where $\lambda_{i}+L_{i}$ denotes $\left(\lambda_{i} I+L\right) / H_{i}$.

Suppose that $\sigma(A) \subset D$ and $(-1)$ is not a proper value of $A$. Then $\sigma(T) \subset D$ and $(-1)$ is not a proper value of $T$, thus $\|T\| \leqq 1$ because $T$ is normal. Thus $T / H_{0}$ satisfies the hypothesis of Theorem 2 , and we conclude that $\left\{B^{n}\right\}$ converges strongly on $H_{0}$. If $\left|\lambda_{i}\right|<1$, then $\sigma\left(\lambda_{i}+L_{i}\right)=\left\{\lambda_{i}\right\}$ is contained in the interior of the unit disc. By Theorem 2 in [11], $\lambda_{i}+L_{i}$ is similar to an operator whose norm is strictly less than 1 . Thus we obtain that $\left\{B^{n}\right\}$ converges uniformly to zero on $H_{i}$, when $\left|\lambda_{i}\right|<1$. Now assume that some $\lambda_{i}=1$. The sequence $\left\{\left(I_{i}+L_{i}\right)^{n}\right\}$ converges weakly since $I_{i}+L_{i}=B / H_{i}$ and $B$ satisfies the hypothesis of Theorem 1 . Since $\left(I_{i}+L_{i}\right)$ is asymptotically bounded, $H_{i}$ is decomposed into the algebraic direct sum $H_{i}=\mathfrak{N}\left(L_{i}\right)+L_{i}\left(H_{i}\right)^{-}$ $[10, \S 144]$. It is clear that on $\Re\left(L_{i}\right),\left\{\left(I_{i}+L_{i}\right)^{n}\right\}$ is strongly convergent. If $x=L_{i}(y)$, then $\left(I_{i}+L_{i}\right)^{n} x=L_{i}\left(I_{i}+L_{i}\right)^{n} y$ is strongly convergent, because $\left\{\left(I_{i}+L_{i}\right)^{n} y\right\}$ is weakly convergent and $L_{i}$ is completely continuous. Hence by Lemma $4,\left\{B^{n}\right\}$ converges strongly on $H_{i}$. Finally if some $\lambda_{i}=-1$, then $H_{i}=L_{i}\left(H_{i}\right)$ - because $(-1)$ is not a proper value of $B$. Just as above, we can conclude that $\left\{B^{n}\right\}$ converges strongly on $H_{i}$ if $\lambda_{i}=-1$. Hence $\left\{B^{n}\right\}$ converges strongly on each $H_{i}(i=0,1, \cdots)$ and thus $\left\{B^{n}\right\}$ is strongly convergent on $H$. Therefore, $A=R^{-1} B R$ is strong asymptotically convergent.

Conversely, if the sequence $\left\{A^{n}\right\}$ converges strongly, then by 
Lemma 2 we can conclude that $\sigma(A) \subset D$ and $(-1)$ is not a proper value of $A$.

We shall now use Theorem 1 in conjunction with the following lemma to prove the strong asymptotic convergence of certain completely continuous operators.

LEMMA 5. If $A$ is a completely continuous operator, then $\left\{A^{n}\right\}$ converges strongly if and only if $\left\{A^{n}\right\}$ converges weakly.

Proof. A completely continuous operator transforms weakly convergent sequences into strongly convergent sequences. Hence if $\left\{A^{n}\right\}$ converges weakly then for $x \in H,\left\{A^{n} x\right\}$ is a weakly convergent sequence so that $\left\{A\left(A^{n} x\right)\right\}=\left\{A^{n+1} x\right\}$ is strongly convergent.

Just as in Theorem 3 , we can prove the strong asymptotic convergence of an operator from the weak asymptotic convergence obtained by applying Theorem 1 to the operator.

Theorem 4. Let $A$ be an asymptotically bounded completely continuous operator. Then $A$ is strong asymptotically convergent if and only if $\sigma(A) \subset D$ and $(-1)$ is not a proper value of $A$.

Proof. Since a completely continuous asymptotically bounded operator is similar to a contraction, as seen in [7], we may assume that $A$ is a contraction. By Lemma $5,\left\{A^{n}\right\}$ converges strongly if and only if $\left\{A^{n}\right\}$ converges weakly. Moreover, by Theorem $1,\left\{A^{n}\right\}$ converges weakly if $\sigma(A) \subset D$ and $(-1)$ is not a proper value of $A$. By Lemma 2, this last statement is also 'only if.'

REMARK. Let $A$ be a contraction which satisfies the hypothesis of Theorem 1, that is, $\sigma(A) \subset D$ and $(-1)$ is not a proper value of $A$. Then if for some integer $k, A^{k}$ satisfies the hypothesis of Theorems 2, 3 , or 4 we can conclude that $\left\{A^{n}\right\}$ converges strongly. This is a consequence of the following lemma.

LEMma 6. Let $A$ be weak asymptotically convergent and for each $x \in H$ there is a sequence of integers $\{n(x)\}$ such that $\left\{A^{n(x)}(x)\right\}$ converges strongly. Then $A$ is strong asymptotically convergent.

Proof. By the Principle of Uniform Boundedness $A$ is asymptotically bounded by $M$. Thus $H=\Re(I-A)+(I-A) H^{-},[10, \S 144]$, where $\Re(I-A)$ and $(I-A) H$ are invariant subspaces of $A$. Clearly if $x \in \Re(I-A)$ then $\left\{A^{n} x\right\}$ converges strongly. If $x \in(I-A) H^{-}$then $\left\{A^{n} x\right\}$ converges weakly to zero, so that $\left\{A^{n(x)} x\right\}$ converges strongly to zero. Now

$$
\left\|A^{i} x\right\|=\left\|A^{k_{i}} A^{n_{i}(x)} x\right\| \leqq\left\|A^{k_{i}}\right\|\left\|A^{n_{i}(x)} x\right\| \leqq M\left\|A^{n_{i}(x)} x\right\|,
$$


where $k_{i}+n_{i}(x)=i$ and $n_{i}(x)$ is the largest element of $\{n(x)\}$ less than or equal to $i$. Since $\left\|A^{n(x)} x\right\| \rightarrow 0$ as $n(x) \rightarrow \infty$ it follows that $\left\|A^{i}(x)\right\|$ $\leqq M\left\|A^{n_{i}(x)} x\right\| \rightarrow 0$ as $i \rightarrow \infty$.

3. Let $T$ be an operator which is strong asymptotically convergent. F. Browder and W. Petryshyn in [3] have shown that this condition enables us to discover solutions of a functional equation of the form

$$
x-T x=y,
$$

by the iteration process of Picard-Poincaré-Neumann. In fact, we define the vectors $x_{n}=T^{n} x_{0}+\left(y+T y+\cdots+T^{n-1} y\right)$, where $x_{0}$ and $y$ are given vectors. Then if $y$ is in the range of $(I-T)$, the sequence $\left\{x_{n}\right\}$ converges strongly to a solution $x$ of $\left(^{*}\right)$.

A similar theorem can be proved for weak asymptotic convergence and applied to contractions $A$ such that $\sigma(A) \subset D$ and $(-1)$ is not a proper value of $A$. The proof presented in [3] for the case of strong asymptotic convergence can be modified in the obvious ways to prove:

THEOREM 5. Let $T$ be a weak asymptotically convergent operator on H. Then:

(a) If $y$ is an element of the range of $(I-T)$, the sequence $\left\{x_{n}\right\}$ defined above for any initial approximation $x_{0}$ will converge weakly to a solution $x$ of $x-T x=y$.

(b) If any subsequence $\left\{x_{n_{j}}\right\}$ of the sequence converges weakly to an element $x$, then $x$ is a solution of $x-T x=y$ and in fact the whole sequence $\left\{x_{n}\right\}$ converges weakly to $x$.

(c) If $\left\{x_{n}\right\}$ is bounded, then $\left\{x_{n}\right\}$ converges weakly to a solution $x$ of $x-T x=y$.

I wish to express my appreciation to Professor Noboru Suzuki for his help and guidance in the preparation of this paper.

\section{REFERENCES}

1. I. Amemeya and T. Ando, Convergence of random products of contractions in Hilbert space, Acta. Sci. Math. 26 (1965), 239-244.

2. M. S. Brodski1 and M. S. Livšic, Spectral analysis of nonselfadjoint operators and intermediate systems, Uspehi Mat. Nauk 13 (1958), 3-85; Amer. Math. Soc. Transl. (2) 13 (1960), 265-346.

3. F. Browder and W. Petryshyn, The solution by iteration of linear functional equations in Banach spaces, Bull. Amer. Math. Soc. 72 (1966), 566-570.

4. N. Dunford, $A$ survey of the theory of spectral operators, Bull. Amer. Math. Soc. 64 (1958), 217-274.

5. S. R. Foguel, Powers of a contraction in Hilbert space, Pacific J. Math. 13 (1963), 551-562. 
6. I. Halperin, The product of projection operators, Acta. Sci. Math. 23 (1962), 96-99.

7. B. Sz.-Nagy, Completely continuous operators with uniformly bounded iterates, Magyar Tud. Akad. Mat. Kutat6 Int. Közl. 4 (1959), 89-93.

8. B. Sz.-Nagy and C. Foias, Analyse harmonique des operateurs de l'espace de Hilbert, Akad. Kiadó, Budapest, 1967.

9. - Sur les contractions de l'espace de Hilbert. IV, Acta Sci. Math. 21 (1960), 251-259.

10. F. Riesz and B. Sz.-Nagy, Functional analysis, Akad. Kiad6, Budapest, 1953.

11. G. C. Rota, On models for linear operators, Comm. Pure Appl. Math. 12 (1960), 469-472.

12. J. Schwartz, On spectral operators in Hilbert space with compact imaginary part, Comm. Pure Appl. Math. 15 (1962), 95-97.

13. N. Suzuki, The structure of spectral operators with completely continuous imaginary part, Proc. Amer. Math. Soc. 22 (1969), 82-84.

University of California, Irvine 